\title{
Equipamento de Monitoramento Contínuo da Floculação (EMCF): experimentação, correlação e calibração
}

\author{
Equipment of Continuous Monitoring Flocculation (ECMF): \\ Experimentation, correlation and calibration.
}

\author{
Data de entrada: \\ 19/12/2014 \\ - Data de aprovação: \\ 25/02/2015
}

\section{Resumo:}

Este artigo visa a aferir e calibrar o sinal $\left(\mathrm{IF}_{\mathrm{EMCF}}\right)$ gerado por um equipamento concebido para monitoramento contínuo da floculação, baseado no princípio das flutuações da turbidez. Para tal, foi empregada técnica não intrusiva e dedicada de análise de imagens e, para aquisição das imagens, foi utilizada câmera Vision Research Miro EX4 acoplada a um conjunto de lentes, permitindo resolução de 840 x 680, com tamanho de pixel de 0,119 $\mathrm{mm}$. O tratamento das imagens foi realizado com auxílio do software Image-Pro Plus. A iluminação utilizada consistiu em plano de laser de $2.000 \mathrm{~mW}$ de potência, com comprimento de onda de $532 \mathrm{~nm}$ e $2 \mathrm{~mm}$ de espessura, ajustado ao foco do sistema de captura de imagens. Os resultados indicaram uma boa explicação dos dados por meio das equações de calibração geradas. 0 maior valor de $\mathrm{R}^{2}$ obtido foi de 0,98 para a calibração do $\mathrm{IF}_{\mathrm{EMCF}}$ por meio do Centro de Massa da Distribuição Volumétrica de Partículas (CMDVP). Tal fato deve-se à maior sensibilidade da flutuação da turbidez para maiores volumes de partículas. Os menores valores de $\mathrm{R}^{2}$ obtidos foram de 0,86 para o diâmetro médio $\left(D_{\text {med }}\right)$ e o Cento de Massa da Distribuição de Tamanho de Partículas (CMDTP). Qualquer que seja o parâmetro desejado, a calibração apresentou valores de erros médios menores que $|7 \%|$. Tais resultados permitem concluir sobre a viabilidade do uso do $\mathrm{IF}_{\mathrm{EMCF}}$ para acompanhamento direto de desempenho da floculação, desde que seja devidamente calibrado para cada característica da água afluente, bem como para os insumos empregados em Estações de Tratamento de Água (ETAs).

Palavras-chave: Floculação. Monitoramento. Calibração.

\section{Rodrigo Braga Moruzzi}

Graduado em Engenharia Civil pela Universidade Federal de São Carlos (UFSCAR). Mestre e doutor em Engenharia Civil na área de Hidráulica e Saneamento pela Universidade de São Paulo (USP). Pós-doutor pela Katholieke Universiteit Leuven. Professor adjunto livre-docente da Universidade Estadual Paulista “Júlio de Mesquita Filho" (Unesp), Campus de Rio Claro.

\section{Andre Luiz de Oliveira}

Graduado em Engenharia Civil pela Universidade Federal de Viçosa (UFV). Mestre e doutor em Engenharia Hidráulica e Saneamento pela USP. Professor doutor da Universidade Federal de Uberlândia - Faculdade de Engenharia Civil.

\section{Marcelo De Julio}

Graduado em Engenharia Civil pela UFSCAR. Especializado em Gestão da Produção pela mesma universidade. Doutor em Engenharia Civil, Área: Hidráulica e Saneamento, pela Escola de Engenharia de São Carlos da Universidade de São Paulo (EESC-USP). Professor adjunto, coordenador do Programa de Pós-Graduação em Engenharia de Infraestrutura Aeronáutica e chefe do Departamento de Recursos Hídricos e Saneamento Ambiental da Divisão de Engenharia Civil do Instituto Tecnológico de Aeronáutica (ITA).

\section{"Endereço para correspondência}

Universidade Estadual Paulista “Júlio de Mesquita Filho", Instituto de Geociências e Ciências Exatas de Rio Claro,

Departamento de Planejamento Regional

Avenida 24 A, 1515 - Bela Vista - Rio Claro, SP-Brasil

$13506-900$ 


\section{Abstract:}

This paper aims to assess and to calibrate the equipment signal $\left(I F_{E M C F}\right)$, based on the principle of turbidity fluctuations, used for continuous monitoring of flocculation. For this purpose, a non-intrusive technique and a dedicated image analysis were applied. For the image acquisition we used a Miro EX4 Vision Research camera coupled to a lens assembly, allowing resolution of $840 \times 680$ with pixel size of $0.119 \mathrm{~mm}$. The Image-Pro Plus software was applied to image treatment. A laser sheet of $2000 \mathrm{~mW}$ of power with wavelength of $532 \mathrm{~nm}$ and 2 $\mathrm{mm}$ thick was adjusted to focus of image capture system. The results indicated a good coefficient of determination for the calibration equations. The highest $R^{2}$ value of 0.98 was obtained from the $I F_{E M C F}$ calibration through the center of mass of the particle volume distribution (PVD CM). This fact is due to the higher sensitivity of the fluctuation of turbidity for larger volumes of particles. The smaller $R^{2}$ values were 0.86 for the medium diameter $\left(D_{\text {med }}\right)$ and the percent mass of the particle size distribution (CMDTP). Whatever the desired parameter, the calibration error values less than $|7 \%|$ were observed. The results indicate that the $I F_{E M C F}$ can be used since it is properly calibrated for water inlet and coagulants used in Water Treatment Plants (WTP).

Keywords: Flocculation. Monitoring. Calibration.

\section{INTRODUÇÃO}

No âmbito dos sistemas de tratamento físico-químico de águas e efluentes, a floculação é a principal etapa responsável por alterar a distribuição e a forma do material particulado, visando à sua posterior remoção em unidades de separação sólido/líquido.

Sob o ponto de vista dos processos e operações da Estação de Tratamento de Água (ETA), constitui ponto nevrálgico no tratamento, pois a característica dos flocos formados tem estreita relação com a característica da água bruta, com os produtos químicos e com a tecnologia empregada. Assim, as características das partículas floculantes são determinantes na eficiência dos processos e na qualidade da água tratada produzida para abastecimento público. Entretanto, os sistemas de tratamento não possuem equipamento capaz de avaliar a distribuição de tamanho de flocos on-line, o que permitiria respostas rápidas e automação do processo. Por outro lado, a Distribuição de Tamanho de Partículas (DTP), a Distribuição Volumétrica de Partículas (DVP) e a morfologia dos flocos fornecem resultados diretos de desem- penho da floculação, omitidos quando utilizadas medidas indiretas, como a medida de turbidez.

O acompanhamento do processo por meio de medidas diretas de desempenho pode ser realizado na câmara de floculação, como apontado por Jarvis et al. (2005). Dessa forma, a DTP, a DVP e o diâmetro médio $\left(D_{\text {med }}\right)$ podem ser aplicados diretamente no controle da coagulação/floculação, isoladamente ou em conjunto, como também observado por Hatukai et al. (1997), Wu e He (2010), Yao et al. (2014), entre outros. Portanto, os resultados apresentados neste artigo visam a aferir e calibrar o sinal gerado por um equipamento concebido para monitoramento contínuo da floculação, baseado no princípio das flutuações da turbidez. A atribuição de significado físico ao sinal gerado permitirá o entendimento do fenômeno e a tomada de decisão em sistemas contínuos.

A principal técnica usada foi inicialmente desenvolvida pela University College London (UCL), por volta de 1984, sendo denominada turbidity fluctuations. Nesse método, uma amostra fluindo em escoamento laminar é submetida à passa- 
gem de feixe de luz por meio de caminho óptico de comprimento conhecido. Tal passagem apresenta variações aleatórias de intensidade na célula receptora. Esse equipamento pode fornecer informações relacionadas ao tamanho do floco durante todo o período de sua formação, quebra e recrescimento, proporcionando informações mais detalhadas de suas propriedades do que as que podem ser obtidas com procedimentos convencionais de monitoramento. A exemplo de um equipamento comercial denominado Photometric Dispersion Analyser (PDA), encontra-se em fase final de desenvolvimento no Brasil, fruto de parceria entre o Instituto Tecnológico de Aeronáutica (ITA), a Companhia de Saneamento Básico do Estado de São Paulo (Sabesp) e a Universidade Estadual Paulista "Júlio de Mesquita Filho" (Unesp), um Equipamento de Monitoramento Contínuo da Floculação (EMCF).

Os princípios fundamentais do monitoramento contínuo da floculação foram previamente discutidos por Gregory e Nelson (1984) e Gregory (1985) e suas aplicações para o monitoramento da floculação, descritas por Brown et al. (1985) e Gregory e Nelson (1986). Resumidamente, a intensidade de um estreito feixe de luz transmitido através de uma suspensão fluindo por um tubo é monitorada por um sensível fotodetector; os resultados consistem em uma componente estável (DC) e uma parte flutuante (AC). DC é simplesmente uma medida da intensidade média de luz transmitida e depende da turbidez (e, portanto, da concentração de sólidos) da suspensão. As flutuações no sinal da luz transmitida surgem de variações aleatórias oriundas da composição da amostra no feixe de luz, a qual é continuamente renovada pelo fluxo (Figura 1). Essas variações originam-se da não uniformidade da suspensão e seguem a distribuição de Poisson (estatisticamente); portanto, o desvio padrão do valor médio é a raiz quadrada do valor médio. Isso implica que o valor médio da raiz quadrada (RMS) desse sinal flutuante depende da raiz quadrada da concentração das partículas.
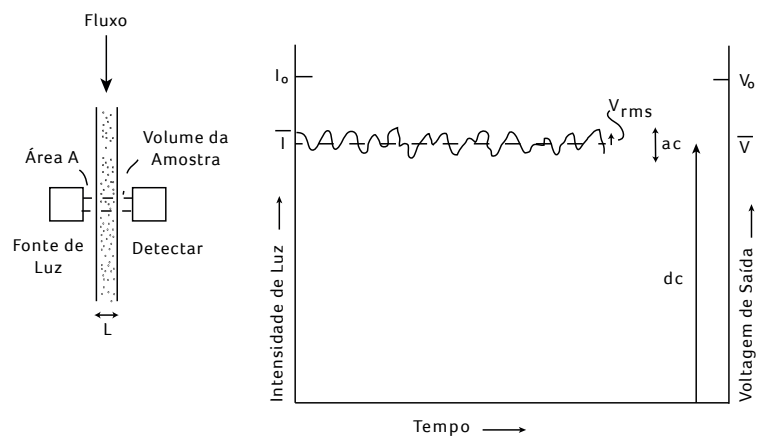

Figura 1 - Flutuações da turbidez em uma dispersão em fluxo.

Fonte: Gregory (1985).

Na prática, é conveniente dividir o valor de RMS por DC para dar uma razão ( $R$ ). Essa medida é adimensional e não é afetada pela contaminação (fouling) das superfícies ópticas ou interferências eletrônicas, podendo ser demonstrado que depende da concentração e tamanho das partículas em suspensão, assim como da dimensão do volume da amostra iluminada. Para o caso geral de uma suspensão heterodispersa, o valor da razão é dado por:

$$
R=(L / A)^{1 / 2}\left(\sum N_{i} C_{i}^{2}\right)^{1 / 2}
$$

Em que: $L$ é o comprimento do caminho óptico; $A$ é a área efetiva da seção transversal do feixe de luz; $N$ é o número de partículas por unidade de volume; e $C$ é a seção transversal de dispersão da partícula. O somatório contempla todos os tipos de partícula $i$, com concentração $N_{i}$ e seção transversal de dispersão da luz $C_{i}$, a qual depende do tamanho e forma das partículas. Cabe mencionar que o valor de $R$ é frequentemente denominado Índice de Floculação (IF).

A Figura 2 apresenta um esquema da célula de fluxo e seus componentes.

Foi verificado que os valores medidos de RMS são independentes da vazão (GREGORY, 1985) e que 


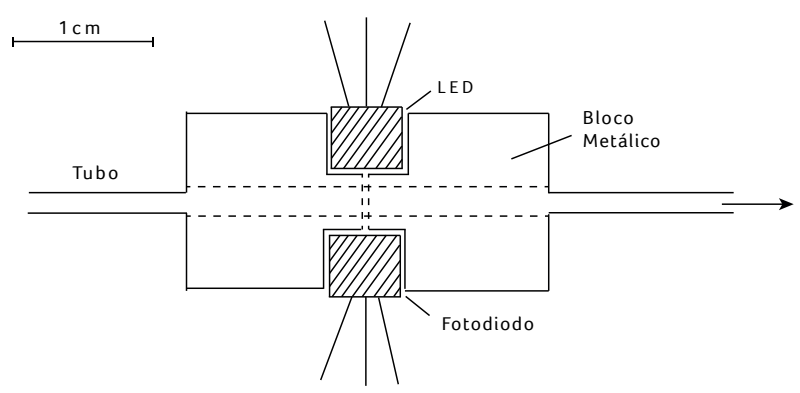

Figura 2 - Esquema da célula de fluxo e dos componentes ópticos.

Fonte: Gregory (1985).

o valor de $R$ fornece uma indicação muito sensível da formação dos agregados (GREGORY; LI, 1991; LI; GREGORY, 1991). Todavia, a aplicação segura da técnica requer a aferição e a calibração do sinal gerado, de modo que a ele possa ser atribuído significado físico, passível de entendimento e que permita tomada de decisão baseada nos fenômenos que governam o processo de floculação. Para tal, são indispensáveis a concepção e a aplicação de método racional que permita a construção de equações que transformem o sinal gerado pelo equipamento (IF) em parâmetros diretos de interesse para o processo de floculação.

Dessa forma, este artigo apresenta os resultados da aplicação de técnica dedicada de análise de imagem, visando à aferição do sinal gerado pelo EMCF, requerendo etapas de experimentação, correlação e calibração.

\section{MATERIAL E MÉTODOS}

A água de estudo, com turbidez de $25 \pm 2$ uT, foi produzida a partir de solução de caulinita, com base no trabalho de Pádua (1994) e de Yukselen e Gregory (2004). O coagulante utilizado foi o sulfato de alumínio $\left[\mathrm{Al}_{2}\left(\mathrm{SO}_{4}\right)_{3} \cdot 14 \mathrm{H}_{2} \mathrm{O}\right.$ ], na dosagem ótima de $2 \mathrm{mgAl}^{+3} / \mathrm{L}$ e pH igual a 7,5, obtidos a partir de diagrama de coagulação/floculação. Foi utilizado EMCF, tendo sido a validação e monitoramento do processo realizados por técnica não intrusiva e dedicada de análise de imagens. Para aquisição das imagens, foi utilizada câmera Vision Research Miro EX4 acoplada a um conjunto de lentes, permitindo resolução de $840 \times 680$, com tamanho de pixel de $0,119 \mathrm{~mm}$. $O$ tratamento das imagens foi realizado com auxílio do software Image-Pro Plus. A iluminação utilizada consistiu em plano de laser de $2.000 \mathrm{~mW}$ de potência, com comprimento de onda de $532 \mathrm{~nm}$ e $2 \mathrm{~mm}$ de espessura, ajustado ao foco do sistema de captura de imagens. A maior vantagem do emprego de método não intrusivo é que as amostras podem ser avaliadas sem risco de deformação da amostragem por ruptura dos flocos.

$\mathrm{Na}$ Figura 3, apresenta-se o aparato experimental: equipamento de bancada jar test acoplado a um sistema não intrusivo de captura de imagens e EMCF, conforme Figura 1.

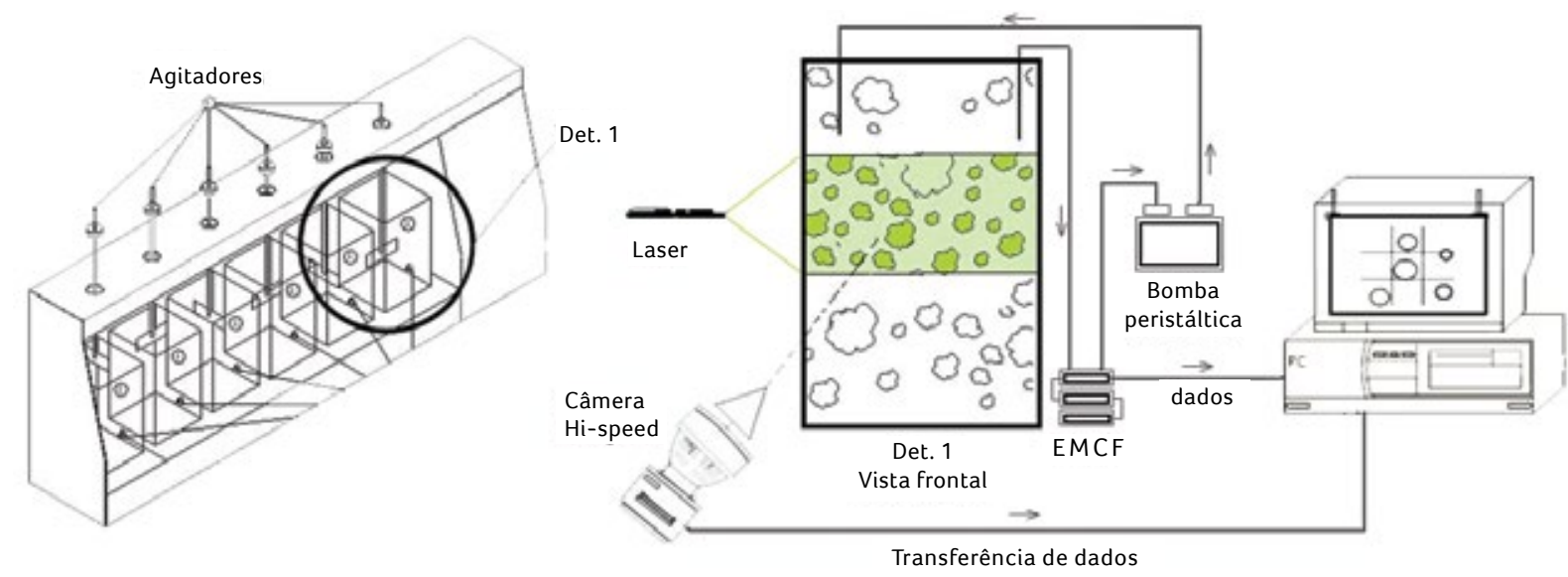

Figura 3 - Aparato experimental (jar test, microcomputador, câmera digital, laser, EMCF, bomba peristáltica). 
Os métodos empregados neste artigo são os mesmos de Moruzzi (2005), apresentados também por Moruzzi e Reali (2007, 2010). Em termos gerais, os passos necessários consistem em: aquisição das imagens; tratamento digital; calibração; determinação das feições de interesse e de tamanho das partículas. Posteriormente, são obtidas as Funções Discretas de Distribuição de Tamanho de Partículas (FDDTPs).

A classificação discreta das partículas, por faixas de tamanho, foi ajustada à forma contínua conforme Equação 2, extraída de Amirtharajah e O’Melia (1999).

$$
\frac{d N}{d\left(d_{p}\right)}=n^{o}\left(d_{p}\right)=A_{o}\left(d_{p}\right)^{-\beta}
$$

Em que: $d N$ é o número de partículas por unidade de volume do fluido na faixa correspondente a $d_{p}$ até $d_{p}+d\left(d_{p}\right) ; d_{p}$ é o diâmetro da partícula; $n^{\circ}\left(d_{p}\right)$ é a função de distribuição de tamanho de partículas; $A_{o}$ é o coeficiente relacionado à concentração total de partículas; $\beta$ é o coeficiente que caracteriza a distribuição de tamanho.

A análise de imagem foi empregada para obtenção de 13 canais (ou classes de tamanho), os quais representam classificações distintas de tamanho, conforme Tabela 1.

O Centro de Massa da Distribuição de Tamanho de Partículas (CMDTP), bem como da distribuição volumétrica (CMDVP), foi determinado a partir da distribuição discreta, conforme Equações 3 e 4. Para obtenção do diâmetro equivalente, definido por Santos et al. (2004), foram identificadas as dimensões de cada partícula definida pela média das dimensões em intervalos de 0 a $360^{\circ}$, com passo de $2^{\circ}$, com referência ao centroide.
Tabela 1 - Classes de tamanho das partículas

\begin{tabular}{|c|c|c|c|}
\hline Classe & $\begin{array}{l}\text { Diâmetro } \\
\text { mínimo } \\
(\mathrm{mm})\end{array}$ & $\begin{array}{l}\text { Diâmetro } \\
\text { máximo } \\
(\mathrm{mm})\end{array}$ & $\underset{(\mathrm{mm})}{d_{p}^{*}}$ \\
\hline 1 & 1 & 125 & 11 \\
\hline 2 & 125 & 250 & 185 \\
\hline 3 & 250 & 375 & 321 \\
\hline 4 & 375 & 500 & 433 \\
\hline 5 & 500 & 625 & 559 \\
\hline 6 & 625 & 750 & 685 \\
\hline 7 & 750 & 875 & 810 \\
\hline 8 & 875 & 1.000 & 935 \\
\hline 9 & 1.000 & 1.125 & 1.061 \\
\hline 10 & 1.125 & 1.250 & 1.186 \\
\hline 11 & 1.250 & 1.375 & 1.311 \\
\hline 12 & 1.375 & 1.500 & 1.436 \\
\hline 13 & 1.500 & 1.625 & 1.561 \\
\hline
\end{tabular}

Nota: * média geométrica da classe.

$$
\begin{aligned}
& C M D T P=\sum_{c=1}^{m} f_{c} \cdot c \\
& C M D V P=\sum_{c=1}^{m} f_{v} \cdot c
\end{aligned}
$$

Em que: $f_{c}$ é a fração de partículas por classe c; $f_{v}$ é a fração em volume de partículas por classe c (o volume é determinado a partir da média geométrica $\left(d_{p}\right)$ do intervalo em cada classe); c é uma das $m$ classes ( $m=13)$, as quais correspondem às faixas de tamanho apresentadas na Tabela 1.

Para verificação das características dos flocos, foram obtidas imagens nos dez segundos finais dos tempos de floculação $\left(T_{f}\right)$ avaliados, a uma taxa de aquisição de $10 \mathrm{~Hz}$. No cômputo global, foram avaliados 375.747 flocos, resultando em erro médio amostral na faixa de $0,5 \%$, para $99 \%$ de confiança. Assim, a função de DTP e seu parâmetro característico $(\beta)$, apresentado na Equação 2 , podem ser empregados para avaliação de todo o conjunto de partículas, uma vez que estas são alteradas a partir das partículas primárias. Faz-se 
necessária, entretanto, a verificação preliminar da sensibilidade do parâmetro $\beta$ em relação a variáveis representativas da DTP.

Foi verificada a sensibilidade do parâmetro $\beta$ frente à variação percentual da primeira classe de tamanho da FDDTP, pois ela está diretamente sujeita às variações advindas da translação das partículas primárias para o limite mínimo (fronteira à esquerda) da DTP, definido pela menor classe de tamanho. Da mesma forma, foi verificada a variável definida pelo CMDTP, pois a mudança de posição do seu valor é indicativa da translação entre as classes de tamanho da FDDTP.

A sensibilidade do parâmetro $\beta$ foi realizada por meio do estudo da correlação múltipla entre as variáveis descritas no parágrafo anterior. Valores do coeficiente de correlação de Pearson $(C P) \geq|0,7|$, para CP $C[-1,1]$, são fortes indícios da representatividade das variações de tamanho de partículas por meio dos parâmetros da função DTP.

Para avaliação da sensibilidade de $\beta$, foram produzidas FDDTPs sintéticas para diferentes classes de tamanho, conservando o intervalo em cada classe e alterando a fração de partículas presentes por classe no sentido das menores para as maiores, tal como ocorre durante o processo de floculação. Foram investigadas correlações múltiplas para três, quatro, cinco, seis, sete, oito, dez e 13 classes de tamanho. Para cada uma das situações produzidas, foram avaliadas as correlações de $\beta$ com o CMDTP e com a variação da primeira classe de tamanho da FDDTP.

\section{RESULTADOS E DISCUSSÃO}

Os resultados de monitoramento contínuo da floculação por meio do EMCF são ilustrados na Figura 4. O tempo de zero minuto representa o instante de lançamento de coagulante. Percebe-se, nitidamente, aumento do sinal (IF) do equipamento em função do tempo de experimento. É conhecido que o aumento do sinal significa aumento das flutuações de turbidez, porém é vital a busca pela sua interpretação por parâmetros representativos da DTP, pois apenas essa conexão pode fornecer sentido físico à medida e, por conseguinte, configurar ferramenta de tomada de decisões. Assim, a obtenção do sinal do EMCF foi acompanhada em tempo real pela aquisição de imagens dos flocos, para futuro tratamento e obtenção das características morfológicas e de tamanho. Conforme ilustra a Figura 4, houve um aumento acentuado no valor do IF entre quatro e sete minutos, acompanhado por perturbações nos valores dos parâmetros avaliados.

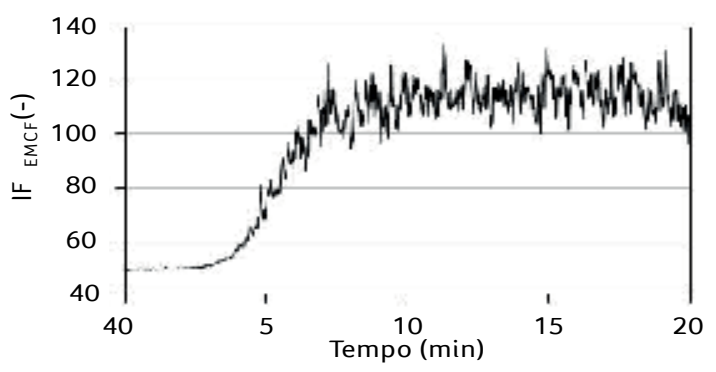

Figura 4 - IF em função do tempo de floculação.

A Figura 5 resulta do acompanhamento do crescimento de flocos por meio de técnica de imagem; os resultados exemplificam a variação do tamanho dos flocos diante do tempo de floculação. A Figura 5a ilustra um conjunto de flocos no início de sua formação, bem como a função discreta de DTP para essa situação; a Figura 5b mostra flocos ainda em formação e a Figura 5c, flocos no patamar de máximo tamanho. As funções discretas de DTP para cada caso registram o avanço do predomínio de partículas de maior tamanho, em detrimento das partículas menores.

A identificação rápida da translação do material particulado para as maiores faixas de tamanho é de fundamental importância no controle da floculação. Isso porque, por reger o sucesso da etapa de tratamento em que se efetiva a separação sólido/ 

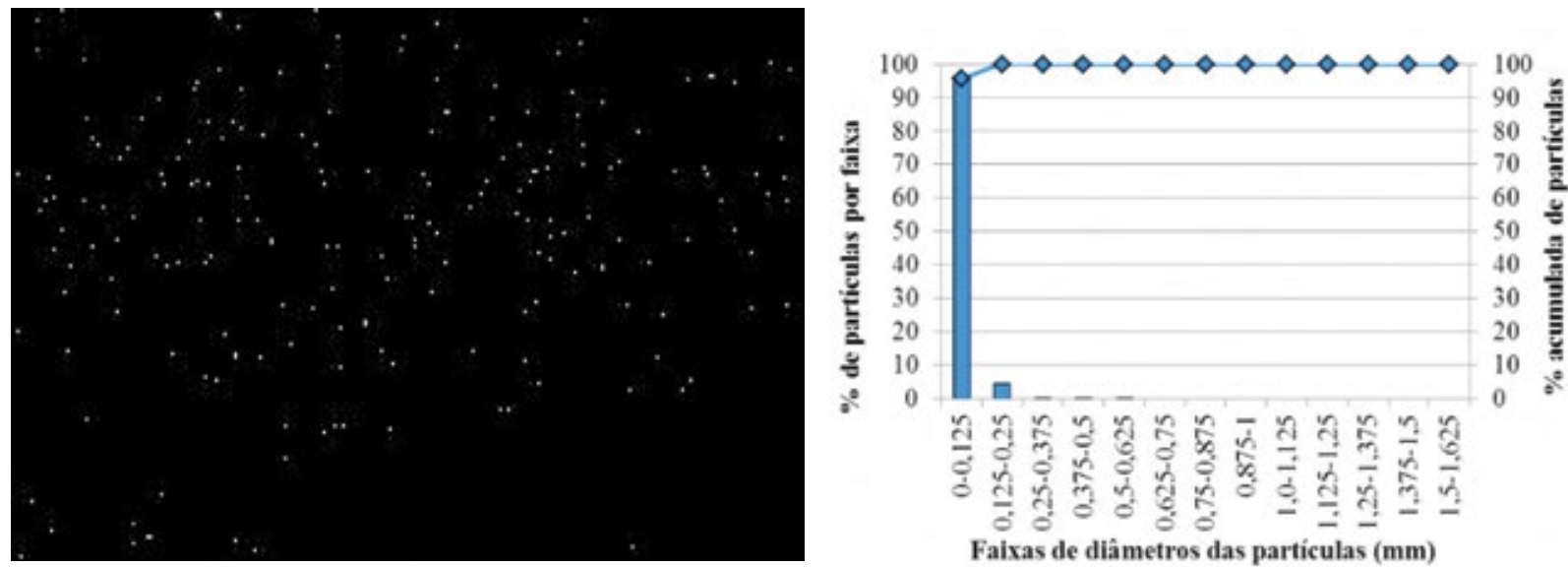

(a) Imagem dos flocos e FDDTP para Tf $=3$ min
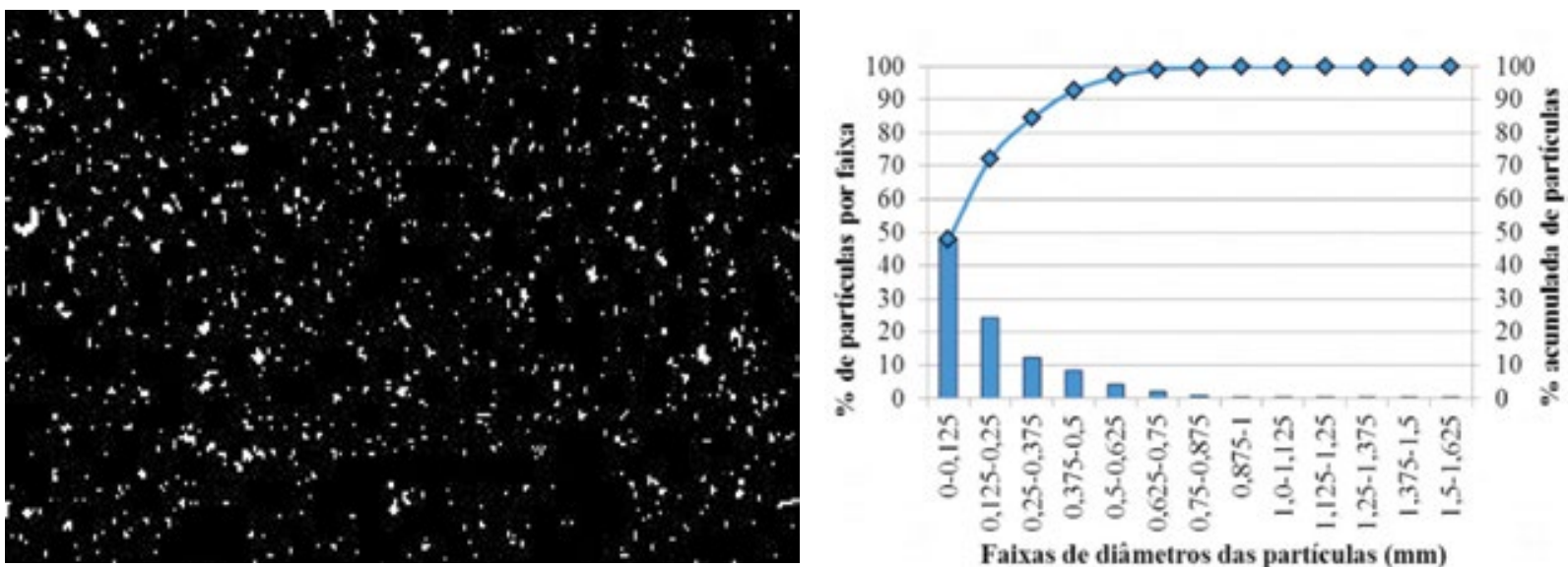

(b) Imagem dos flocos e FDDTP para $\mathrm{T}_{\mathrm{f}}=6 \mathrm{~min}$
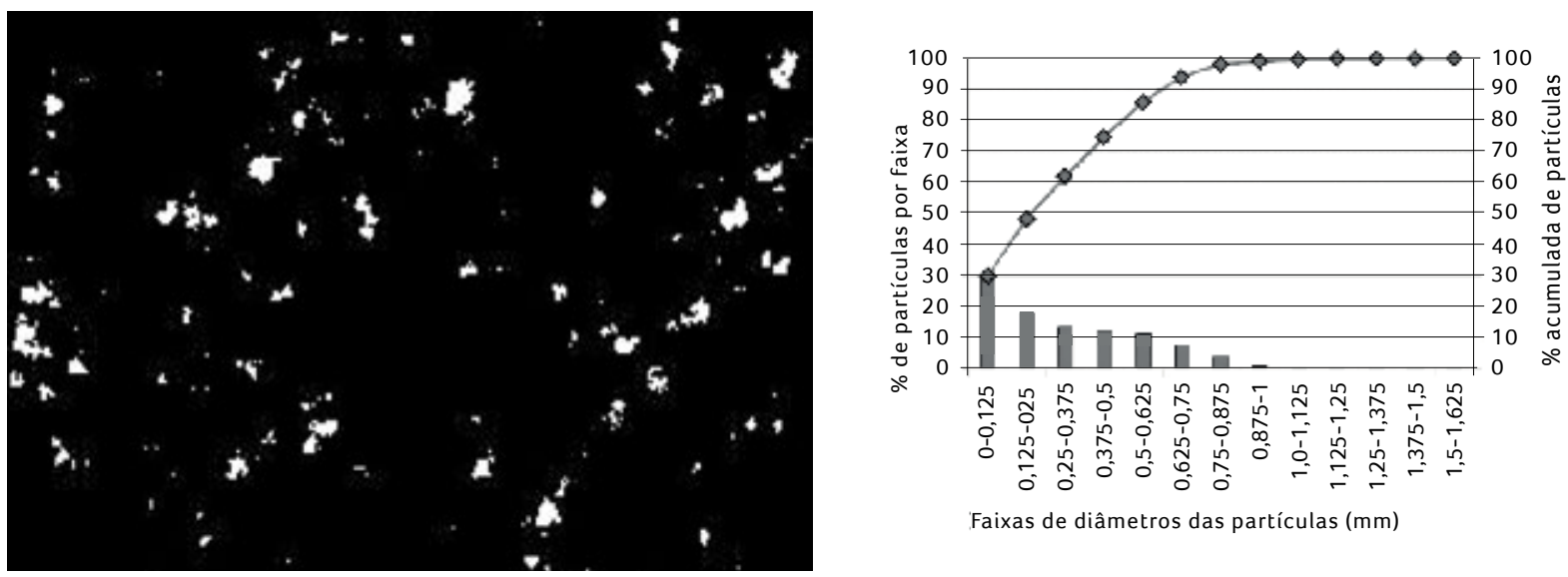

Faixas de diâmetros das partículas (mm)

(c) Imagem dos flocos FDDTP para $\mathrm{T}_{\mathrm{f}}=15 \mathrm{~min}$

Figura 5 - Imagens dos flocos a diferentes tempos de floculação e representação gráfica das respectivas FDDTPs. 
líquido, a floculação é o principal alvo de controle dos sistemas. A obtenção on-line do IF, que descreve as perturbações em uma suspensão, e sua correlação com suas características morfológicas e de tamanho conferem sentido físico ao sinal e possibilitam sua utilização como ferramenta de tomada de decisão. Para dar prosseguimento à aferição, foi verificada a sensibilidade do parâmetro da função contínua de DTP, conforme Equação 2 , e os resultados, obtidos por meio da FDDTP. $\mathrm{Na}$ Tabela 2, podem-se verificar claramente fortes correlações entre o parâmetro característico da função contínua $(\beta)$ e as distribuições geradas sinteticamente (correlações $\geq|0,89|$ ). Como esperado, as variações da primeira classe de tamanho respondem em razão inversamente proporcional ao $\beta$, enquanto as variações do CMDTP respondem em razão direta.

Esse conjunto de resultados, explicitados pelas correlações entre as variáveis e suas relações em termos absolutos, permitiu inferir sobre o potencial de representação das oscilações da FDDTP, presentes durante o processo de floculação, por meio do parâmetro característico $\beta$ da Função Contínua de Distribuição de Tamanho de Partícula (FCDTP) para uma faixa ampla de classes de tamanho (de três a 13 classes), mantendo-se o intervalo em cada classe. Tal constatação possibilitou a continuidade dos trabalhos, no que tange às atividades subsequentes, visando à calibração do sinal gerado pelo equipamento (IF) e ao significado físico expresso pelos parâmetros da FCDTP.

Tabela 2 - Correlações lineares múltiplas entre o parâmetro característico da distribuição de tamanho $(\beta)$, o centro de massa da distribuição de tamanho de partículas $(C M)$ e a primeira classe relativa à distribuição de tamanho de partículas (1 ${ }^{\text {a }}$ Classe (\%)). Distribuições sintéticas geradas para um total de 3, 4, 5, 6, 7, 8, 10 e 13 classes de tamanho.

\begin{tabular}{|c|c|c|c|c|c|c|c|}
\hline Número de Classes & 3 & & & Número de Classes & 7 & & \\
\hline & $\beta$ & CMDTP & $1^{\mathrm{a}}$ Classe & & $\beta$ & CMDTP & $1^{\mathrm{a}}$ Classe \\
\hline$\beta$ & 1 & & & $\beta$ & 1 & & \\
\hline CMDTP & 0,96 & 1 & & CMDTP & 0,95 & 1 & \\
\hline $1^{\text {a }}$ Classe & $-0,95$ & $-0,99$ & 1 & $1^{\mathrm{a}}$ Classe & $-0,96$ & $-0,99$ & 1 \\
\hline \multirow[t]{2}{*}{ Número de Classes } & 4 & & & Número de Classes & 8 & & \\
\hline & $\beta$ & CMDTP & $1^{\mathrm{a}}$ Classe & & $\beta$ & CMDTP & $1^{\mathrm{a}}$ Classe \\
\hline$\beta$ & 1 & & & $\beta$ & 1 & & \\
\hline CMDTP & 0,99 & 1 & & CMDTP & 0,92 & 1 & \\
\hline $1^{\mathrm{a}}$ Classe & $-0,99$ & $-0,99$ & 1 & $1^{\mathrm{a}}$ Classe & $-0,95$ & $-0,95$ & 1 \\
\hline \multirow[t]{2}{*}{ Número de Classes } & 5 & & & Número de Classes & 10 & & \\
\hline & $\beta$ & CMDTP & $1^{\mathrm{a}}$ Classe & & $\beta$ & CMDTP & $1^{\mathrm{a}}$ Classe \\
\hline$\beta$ & 1 & & & $\beta$ & 1 & & \\
\hline CMDTP & 0,95 & 1 & & CMDTP & 0,99 & 1 & \\
\hline $1^{\mathrm{a}}$ Classe & $-0,97$ & $-0,99$ & 1 & $1^{\mathrm{a}}$ Classe & $-0,95$ & $-0,97$ & 1 \\
\hline \multirow[t]{2}{*}{ Número de Classes } & 6 & & & Número de Classes & 13 & & \\
\hline & $\beta$ & CMDTP & $1^{\mathrm{a}}$ Classe & & $\beta$ & CMDTP & $1^{\text {a }}$ Classe \\
\hline$\beta$ & 1 & & & $\beta$ & 1 & & \\
\hline CMDTP & 0,97 & 1 & & CMDTP & 0,99 & 1 & \\
\hline $1^{\mathrm{a}}$ Classe & $-0,96$ & $-0,99$ & 1 & $1^{\mathrm{a}}$ Classe & $-0,90$ & $-0,89$ & 1 \\
\hline
\end{tabular}


$\mathrm{Na}$ Tabela 3, apresentam-se as correlações obtidas entre o sinal do equipamento $\left(\mathrm{IF}_{\mathrm{EMCF}}\right)$ e os atributos físicos das partículas, dados por: $\beta, D_{\text {med }}$, CMDTP e CMDVP. Foram observadas fortes correlações entre os parâmetros mencionados, indicativas da

Tabela 3 - Correlações lineares múltiplas entre IF $_{\text {EMCF}}$, $\beta, D_{\text {med }}$, CMDTP e CMDVP.

\begin{tabular}{|l|c|c|c|c|c|}
\hline & $\mathrm{I}_{\text {EMCF }}$ & $\beta$ & $D_{\text {med }}$ & CMDTP & CMDVP \\
\hline $\mathrm{IF}_{\text {EMCF }}$ & 1,000 & & & & \\
\hline$\beta$ & $-0,961$ & 1,000 & & & \\
\hline$D_{\text {med }}$ & 0,930 & $-0,883$ & 1,000 & & \\
\hline CMDTP & 0,926 & $-0,880$ & 0,999 & 1,000 & \\
\hline CMDVP & 0,990 & $-0,947$ & 0,900 & 0,895 & 1,000 \\
\hline
\end{tabular}
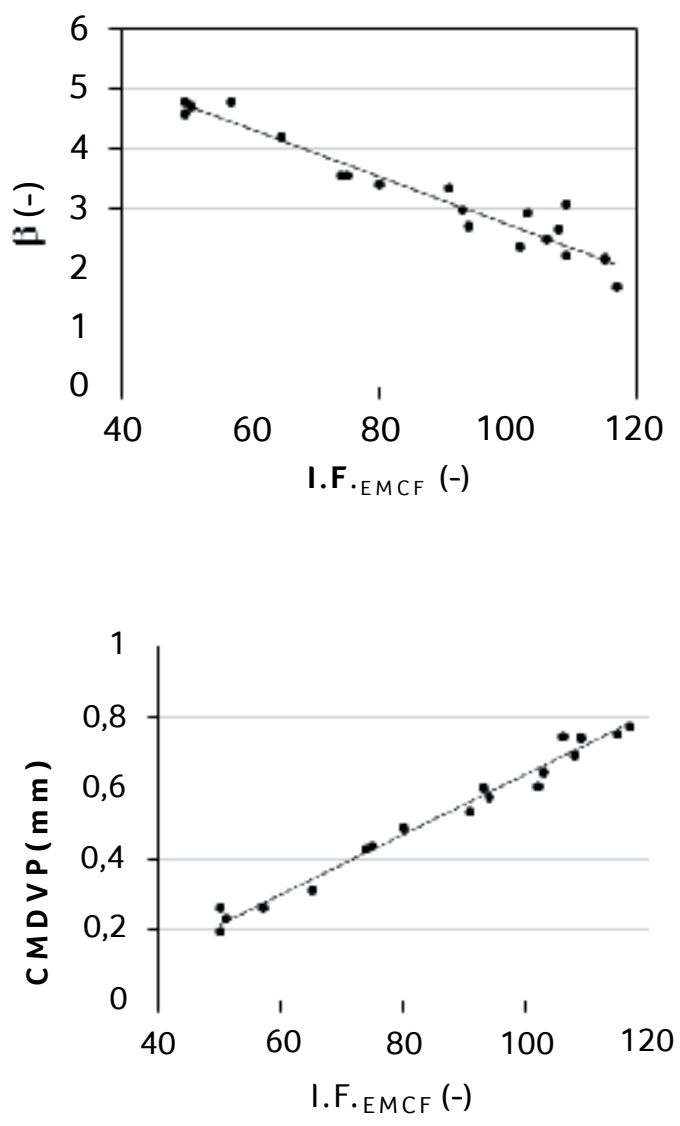

excelente tradução do sinal do equipamento pelo significado físico correspondente, implementado por meio da calibração.

Foi observada correlação inversa de $\beta$ com os demais parâmetros avaliados. De fato, conforme descrito por Crittenden et al. (2005), o valor negativo representa o domínio de partículas maiores, enquanto o valor positivo indicaria domínio das partículas de menor tamanho. Valor de $\beta$ igual à unidade indicaria distribuição homogênea.

Os resultados compilados na Figura 6 referem-se às curvas de calibração do $\mathrm{IF}_{\mathrm{EMCF}}$ com os parâmetros físicos. Em complemento, são apresentados na Tabela 4 os coeficientes de explicação do ajus-
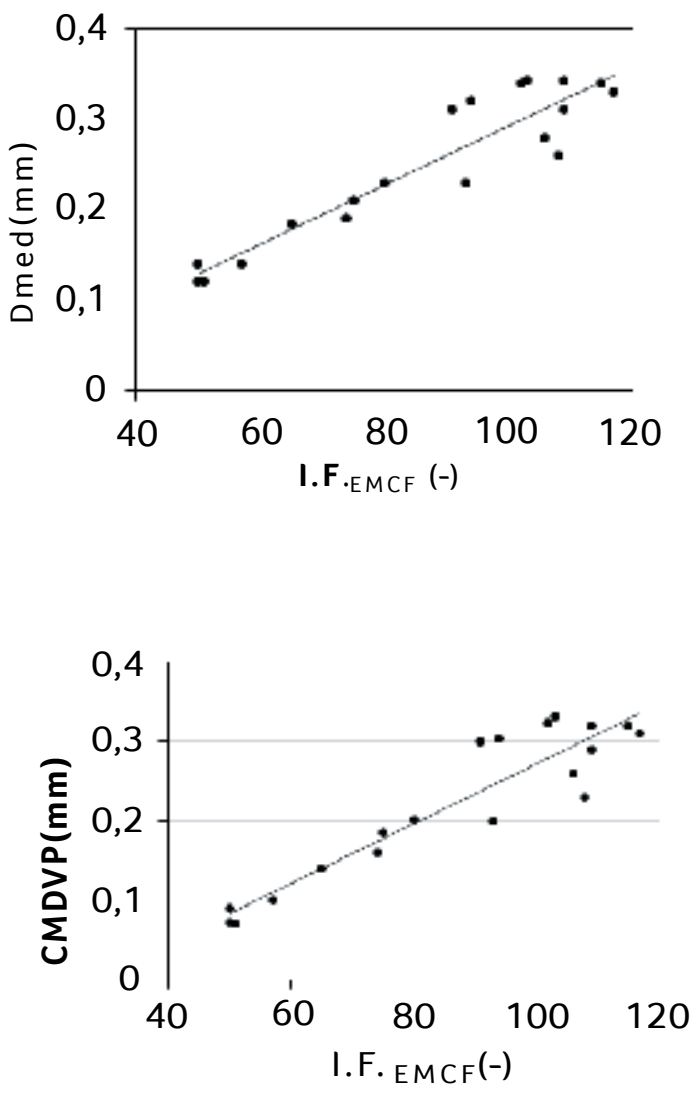

Figura 6 - Curvas de calibração do $I F_{E M C F}$ com os parâmetros de significado físico.

Nota: O símbolo (-) indica variável adimensional. 
te por regressão linear $\left(R^{2}\right)$, bem como as equações de conversão geradas para o sinal obtido e os erros médios estimados entre os parâmetros medidos e calculados.

Os valores obtidos de $\mathrm{R}^{2}$ indicam uma boa explicação dos dados por meio das equações de calibração geradas. $\mathrm{O}$ maior valor de $\mathrm{R}^{2}$ obtido foi de 0,98 para a calibração do sinal do $\mathrm{IF}_{\mathrm{EMCF}}$ por meio do CMDVP. Tal fato deve-se à maior sensibilidade da flutuação da turbidez para maiores volumes de partículas. O menor valor de $\mathrm{R}^{2}$ obtido foi de 0,86 para os parâmetros $D_{\text {med }}$ e CMDTP. Nesse caso, atribui-se à interferência do número de partículas presentes nas menores classes de tamanho. Cabe ser mencionado que o ajuste por regressão do parâmetro $\beta$ obteve valor de $\mathrm{R}^{2}$ de 0,93 , mas esse parâmetro oscilou em faixa mais estreita quando comparado aos demais, como pode ser visualizado na distribuição quartil padronizada dos parâmetros apresentada na Figura 7. Essa constatação é forte indicativa da sensibilidade associada à precisão, o que torna o parâmetro $\beta$ bom indicador direto de variação. Todavia, qualquer que seja o parâmetro desejado, a calibração apresentou valores de erros médios menores que $|7 \%|$, dentro de faixas de equipamentos comerciais tradicionalmente empregados.

Tais resultados permitem concluir sobre a viabilidade do uso do EMCF para acompanhamento direto de desempenho da floculação, desde que

Tabela 4 - Correlações lineares múltiplas entre IF $_{\text {EMCF}}$, $\beta, D_{\text {med }}$, CMDTP e CMDVP e erros médios estimados.

\begin{tabular}{|c|c|c|}
\hline Curva de calibração & $\mathbf{R}^{2}$ & Erro (\%) \\
\hline$\beta=-0,0401 \times I . F_{\text {EMCF }}+6,7605$ & 0,93 & -7 \\
\hline$D_{\text {med }}=0,0033 \times I . F_{\text {EMCF }}-0,0376$ & 0,86 & 5 \\
\hline$C M D T P=0,0038 \times I . F_{\text {EMCF }}-0,106$ & 0,86 & 4 \\
\hline$C M D V P=0,0084 \times I . F_{\text {EMCF }}-0,202$ & 0,98 & 2 \\
\hline
\end{tabular}

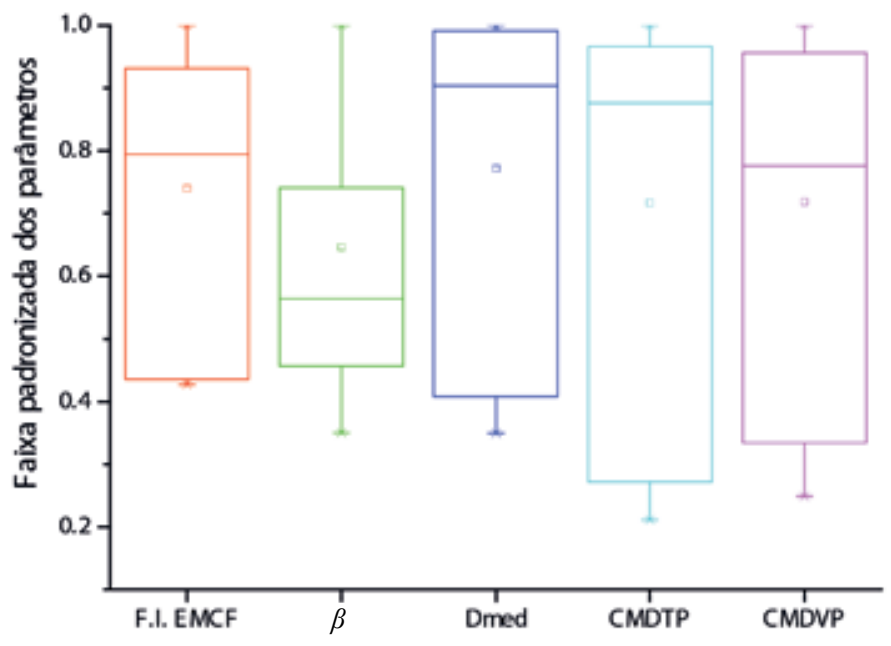

Figura 7 - Distribuições quartis padronizadas dos parâmetros de medida direta de partículas 
seja devidamente calibrado para cada característica da água afluente, bem como para os insumos empregados em ETAs. Os resultados apresentados, com ênfase no uso do equipamento e na atribuição de significado físico ao sinal gerado, abrem uma janela de possibilidades para a automação de processos, com destaque para sistemas que empregam a floculação como uma das etapas de tratamento.

Com a finalidade de ilustrar a aplicação do sinal calibrado, proveniente do equipamento mencionado, pode-se supor, a título de exemplo, uma ETA hipotética em ciclo completo com um conjunto de quatro câmaras de floculação interligadas a um decantador convencional com três unidades de filtração subsequentes. Suponha-se que a qualidade da água do manancial varie na época de seca e de chuva e que em cada época seja requerido um determinado desempenho dos floculadores, de modo que o sistema possa operar com ciclo completo ou com filtração direta descendente. Suponha-se também, nesse exemplo, que a boa prática operacional permita determinar as condições demandadas em cada caso, inclusive definindo o $D_{\text {med }}$ requerido na saída de cada câmara, de modo a atingir a eficiência necessária ao final do sistema.

Assim, a variação sazonal sem alteração da vazão afluente à ETA demanda o rápido ajuste da rotação dos agitadores, de modo a promover o gradiente médio de velocidade $\left(G_{f}\right)$ adequado. Tal procedimento deve ser feito à luz de resultados preliminares, definidos por ensaios dedicados, mas pode ser acompanhado de perto e on-line por meio do sinal gerado e de sua relação com o $D_{\text {med }}$.

Supõe-se que o valor de $D_{\text {med }}$ adequado para operação do sistema prescindindo o decantador seja de $130 \mathrm{~mm}$ na saída da primeira câmara ( $\mathrm{IF}_{\mathrm{EMCF}}$ de 51), de $270 \mathrm{~mm}$ na saída da segunda câmara $\left(\mathrm{IF}_{\mathrm{EMCF}}\right.$ de 93), de $310 \mathrm{~mm}$ na saída da terceira câmara $\left(\mathrm{IF}_{\mathrm{EMCF}}\right.$ de 105) e de $350 \mathrm{~mm}$ na saída da quarta câmara $\left(\mathrm{IF}_{\mathrm{EMCF}}\right.$ de 118). Medidas in loco indicam que a saída na terceira câmara é de 305 mm, inferior ao desejado. As mesmas medidas apontam que a primeira e segunda câmaras operam adequadamente, com saídas de $D_{\text {med }}$ dentro dos valores requeridos. Sendo assim, determina-se com precisão que atenção corretiva deve ser dada à terceira câmara de floculação.

Quando uma das câmaras não atinge o valor de $D_{\text {med }}$ requerido para o alcance da eficiência global, pode-se conferir a rotação imprimida no agitador. Caso esta esteja adequada, pode-se, por exemplo, detectar algum problema nas pás dos agitadores, dificilmente visualizado quando da operação do sistema. Assim, quando o valor do $D_{\text {med }}$ requerido não é atingido, podem-se diagnosticar e propor rápidas alterações, antes mesmo que o sistema comece a produzir água tratada com qualidade indesejada, a depender da natureza e extensão do problema.

Evidentemente, trata-se de exemplo ilustrativo que abrange somente uma das possibilidades da aplicação dos resultados deste artigo, os quais extrapolam a simples medida do sinal gerado. Existem, no entanto, aplicações que vão muito além das mencionadas e que podem ser conjugadas à operação de sistemas de tratamento quando o sinal do EMCF é adequadamente calibrado.

\section{CONCLUSÃO}

Os resultados apresentados neste artigo permitem concluir que o sinal gerado pelo EMCF é adequado às variações de tamanho de flocos e que, quando devidamente calibrado, pode ser empregado para acompanhamento do desempenho da floculação em sistemas contínuos.

Ressalta-se que a calibração e aferição do equipamento são imprescindíveis para a boa resposta do sistema, devendo ser realizadas para cada característica da água bruta afluente à ETA, bem como para cada coagulante empregado no sistema de tratamento. 


\section{AGRADECIMENTOS}

Os autores agradecem à Fundação de Amparo à Pesquisa do Estado de São Paulo (Fapesp) eà Companhia de Saneamento Básico do Estado de São Paulo (Sabesp) pelo apoio financeiro (Processos FAPESP 2010/50694-0, 2013/21355-1, 2013/01634-3 e 2013/25641-9), ao ITA e à Unesp, Campus de Rio Claro, pela infraestrutura disponibilizada.

\section{REFERÊNCIAS}

AMIRTHARAJAH, A.; O'MELIA, C.R. (1999). Coagulation Processes: Destabilization, Mixing, and Flocculation. In: AWWA. Water quality and treatment - A handbook of community water supplies. (Chapter 6). McGraw-Hill, Inc., 5th ed. USA, 1233p, 1999.

BROWN, G.M.; GREGORY, J.; JACKSON, P.J.; NELSON, D.W.; TOMLINSON, E.J. (1985). An on-line monitor for flocculation control. In: Instrumentation and Control of Water and Wastewater Treatment and Transport Systems (Edited by Drake R. A. R.), Pergamon Press, Oxford, p.239-245.

CRITTENDEN, J. C; TRUSSELL, R. R; HAND, D. W, HOWE, K. J.; TCHOBANOGLOUS, G. (2005) Water Treatment: Principles and Design. 2nd ed.

GREGORY, J. (1985). Turbidity fluctuations in flowing suspensions. Journal of Colloid and Interface Science, v.105, n.2, p.357-371, June.

GREGORY, J.; LI, G. (1991). Effects of dosing and mixing conditions on polymer flocculation of concentrated suspensions. Chem. Eng. Comm. Gordon and Breach Science Publishers S.A., Printed in the USA, v.108, p.3-21.

GREGORY, J.; NELSON, D.W. (1984). A new optical method for flocculation monitoring. In: J. Gregory (Ed.), Solid-Liquid Separation, Ellis Horwood, Chichester, p. 172-182.

GREGORY, J.; NELSON, D.W. (1986). Monitoring of aggregates in flowing suspensions. Colloids and Surfaces, 18, p.175-188.

HATUKAI, S.; BEN-TZUR, Y.; REBHUN, M. (1997) Particle counts and size distribution in system design for removal of turbidity by granular deep bed filtration. Wat. Sci. Tech., 36 (4), 225-230.
JARVIS, P.; JEFFERSONA, B.; GREGORY, J.; PARSONS, S. A. (2005) $A$ review of floc strength and breakage. Water Research 39, 3121-3137.

LI, G.; GREGORY, J. (1991). Flocculation and sedimentation of high-turbidity waters. Water Research, Pergamon Press plc, Printed in Great Britain, v.25, n.9, p.1137-1143.

MORUZZI, R. B. (2005). Avaliação da Influência da Distribuição de Tamanho de Partículas e do Binômio Velocidade/Tempo de Detenção na Zona de Reação no Desempenho da Flotação com Utilização de Sonda Ultrasônica e Técnica de Análise por Imagem. Tese de doutorado apresentada à Escola de Engenharia de São Carlos, Universidade de São Paulo. São Carlos: EESC - USP. 240p.

MORUZZI, R.B.; REALI, M.A.P. (2007). Método para determinação de distribuição de tamanho de microbolhas (DTMB) em sistemas de flotação (FAD) para tratamento de águas utilizando a análise de imagem digital. Revista Engenharia Sanitária e Ambiental, Rio de Janeiro, 12, (3), p. 273-283.

MORUZZI, R.B.; REALI, M.A.P. (2010). Characterization of micro-bubble size distribution and flow configuration in DAF contact zone by anon-intrusive image analysis system and tracer tests. IWA Publishing, Water Science \& Technology, 61, (1), p.253-262.

PÁDUA, V.L. (1994) Metodologia para determinação dos gradientes de velocidade médios em unidades de floculação de mistura completa com câmaras em série e escoamento contínuo a partir de reatores estáticos. 165 p. São Carlos. Dissertação (Mestrado em Hidráulica e Saneamento). Escola de Engenharia de São Carlos, Universidade de São Paulo.

SANTOS, H. R.; PRADO, G. S.; VIDAL, C. M. S.; MORUZZI, R. B. \& CAMPOS, J. R. Aplicabilidade das técnicas de determinação de tamanho de partículas em sistemas de tratamento de água e esgoto sanitário. Revista Brasileira de Engenharia Sanitária e Ambiental, v. 9, n. 4, p. $291-300,2004$

WU, J. \& HE, C. (2010) Experimental and modeling investigation of sewage solids sedimentation based on particle size distribution and fractal dimensión. Int. J. Environ. Sci. Tech., 7 (1), 37-46.

YAO, M.; NAN, J.; CHEN, T. (2014) Effect of particle size distribution on turbidity under various water quality levels during flocculation processes. Desalination, 354, 116-124.

YUKSELEN, M. A., AND GREGORY, J. (2004) The reversibility of flocs breakage. Int. J. Miner. Process., 73, 251-259. 